\title{
How to maintain nutritional status after esophagectomy via the Tanaka method
}

\author{
Yoshihiro Tanaka, Yuta Sato, Tomonari Suetsugu, Junichi Mase, Ritsuki Takaha, Naoki Okumura, \\ Nobuhisa Matsuhashi, Takao Takahashi, Kazuhiro Yoshida \\ Department of Surgical Oncology, Graduate School of Medicine, Gifu University, Gifu 501-1194, Japan.
}

Correspondence to: Dr. Yoshihiro Tanaka, Department of Surgical Oncology, Graduate School of Medicine, Gifu University, 1-1 Yanagido, Gifu 501-1194, Japan. E-mail: yoshihirotana11@hotmail.com

\begin{abstract}
How to cite this article: Tanaka Y, Sato Y, Suetsugu T, Mase J, Takaha R, Okumura N, Matsuhashi N, Takahashi T, Yoshida K. How to maintain nutritional status after esophagectomy via the Tanaka method. J Cancer Metastasis Treat 2021;7:59.

https://dx.doi.org/10.20517/2394-4722.2021.151
\end{abstract}

Received: 14 Jul 2021 First Decision: 10 Sep 2021 Revised: 11 Sep 2021 Accepted: 28 Sep 2021 Published: 21 Oct 2021

Academic Editors: Lucio Miele, Giovanni Tuccari, Masayuki Watanabe Copy Editor: Xi-Jun Chen Production Editor: Xi-Jun Chen

\begin{abstract}
Aim: Many therapeutic means have emerged to treat esophageal cancer. Factors relating to nutritional status such as body weight maintenance are important to the continuation of these treatments. In this study, we investigated methods to extend the administration of diversified treatments for patients with esophageal cancer, especially regarding measures to suppress body weight loss after surgery.
\end{abstract}

Methods: We retrospectively evaluated a strategy for preventing postoperative body weight loss which can hinder the continuation of treatment via a reconstruction method aimed at safety and comfort combined with postoperative dietary intake and nutritional support for esophageal cancer patients.

Results: The subjects comprised 386 patients who underwent subtotal stomach reconstruction during esophageal cancer surgery performed from January 2008 to January 2021 at Gifu University Hospital. The anastomotic leakage rate was $0.5 \%$. By administering oral nutritional supplementation under strict rules using ENSURE ${ }^{\circledR} H$ during the perioperative period, the percentage of body weight loss after 5 years could be limited to $4.78 \%$ compared to that before treatment. Scores assessing early feeling of fullness measured using the EORTC QLQ-OES18 at postoperative months $3,12,24,36,48$, and 60 were $1.7 \pm 0.3,2.0 \pm 0.2,1.2 \pm 0.3,1.3 \pm 0.2,1.5 \pm 0.2$, and $1.4 \pm 0.1$, respectively. 
Conclusion: Considering methods to eliminate the factors that prevent continuation of treatment may lead to sustainable treatment against esophageal cancer. Proper surgical reconstruction and nutritional management will allow maintenance of body weight and good quality of life.

Keywords: Esophageal cancer, nutrition, reconstruction, oral nutritional supplementation

\section{INTRODUCTION}

Among the number of cases of cancer predicted to occur in Japan in 2020, that of esophageal cancer (EC) was the 11 th highest, and the number of deaths was predicted to be 11,100 per year, which is the 10th highest rate ${ }^{[1]}$. EC continues to have a poor prognosis, with 5 -year survival rates ranging from $20 \%$ to $35 \%$ for the non-metastatic form ${ }^{[2]}$. Survival is dependent on the stage of disease, with 5 -year relative overall survival of only $3.1 \%$ for metastatic disease ${ }^{[3]}$. Currently, endoscopic resection for early EC, minimally invasive esophagectomy, chemoradiotherapy, neoadjuvant or induction chemotherapy ${ }^{[4-7]}$, and immune checkpoint inhibitors are reported to be effective for EC patients ${ }^{[8]}$. With the advent of immune checkpoint inhibitors potentially improving the treatment outcomes for EC, it has now become important to eliminate as much as possible events related to body weight loss and poor quality of life as well as adverse events during treatment for $\mathrm{EC}^{[9-11]}$.

The functional role of the surgically reconstructed stomach is important given that it is an early route for food intake, which is its physiological role. Surgeons need to aim for reconstruction that is safe and does not interfere with postoperative dietary intake, allowing for supplementation against the lack of calories with enteral nutritional supplements.

Therefore, this paper focuses on the measures thought to be necessary for patients to continue receiving cancer treatment, and it retrospectively considers how to create a gastric tube that causes no postoperative difficulty in eating and how to prevent body weight loss using oral nutritional supplementation (ONS).

\section{METHODS}

\section{Surgical reconstruction to prevent postoperative difficulty in eating}

In a previous report, we described subtotal gastric reconstruction in detail ${ }^{[12]}$. To improve the technique over the last few years, we have been devising ways to eliminate food stagnation. After creating a hand-sewn anastomosis at the neck, we first pull the elevated stomach caudally, and second, we especially pull the greater curvature side of the stomach to the caudal side to linearize the stomach in the thoracic cavity. Figure 1A shows the cut line for creation of the subtotal stomach (SS). After creating an anastomosis at the cervical wound, we pull in the direction of the blue arrow to eliminate the bulge peculiar to the SS, as indicated by the red dashed arrows in Figure 1B. Next, a deflection of the SS is present above the diaphragm especially at the greater curvature side, as indicated by the red dashed arrow in Figure $1 \mathrm{C}$, and we pull in the direction of the blue arrow to eliminate it. Currently, the number of stiches used to fix the SS to the diaphragmatic leg has been increased from 3 to 7 [Figure 1D], because the greater curvature side of the stomach may be pulled into the thoracic cavity after surgery, and food flow may stagnate in the diaphragmatic leg. Increasing the number of stiches prevents this occurrence. Finally, we performed pyloroplasty by the finger fracture method.

\section{Combined use of ONS based on blood glucose level and appetite for 6 months postoperatively}

We have been actively using enteral nutrition administered via jejunostomy tube or orally from the perioperative period to about 6 months after surgery. ENSURE ${ }^{\oplus} \mathrm{H}$ (Abbott Japan LLC) at a concentration of 

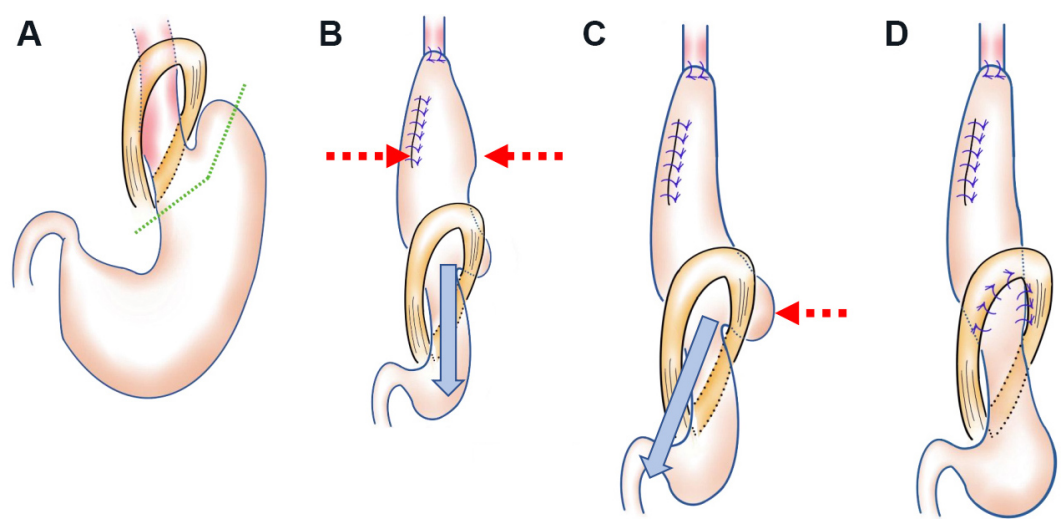

Figure 1. Creating subtotal stomach reconstruction. (A) Cut line of the stomach. (B) Pull in the direction of the blue arrow to eliminate the bulge peculiar to the stomach, as indicated by the red dashed arrows. (C) Deflection of the stomach is present above the diaphragm especially at the greater curvature side, as indicated by the red dashed arrow, so we pull in the direction of the blue arrow to eliminate it. (D) The number of stiches used to fix the stomach to the diaphragmatic leg should be seven.

$1.5 \mathrm{kcal} / \mathrm{mL}$ is being used. In particular, we have focused on the fact that a vague all-day rise in blood sugar level reduces appetite. Enteral nutritional supplementation is started the day after surgery, and calories are gradually increased. At the stage when oral intake became stable in the period before discharge, enteral nutrition is administered via jejunostomy tube or orally twice a day ( $375 \mathrm{kcal}$ each) at the same time as breakfast and dinner, and an administration time of $3 \mathrm{~h}$ at each meal is strictly adhered to. This method of ingesting ONS is called the Tanaka method (taking an ONS along with the meal by knowing appetite), and ONS is used for 6 months after EC surgery. Figure 2 shows an example of three timings at which ONS can be taken twice daily for $3 \mathrm{~h}$ each. (1) Taking ONS between meals: When the blood glucose level after breakfast/lunch rose to its highest level, ONS was taken orally, but compliance did not improve. Patients complained that they could not take ONS due to feeling full. They complained of feeling like they were eating all day long and that their daily activities were restricted. (2) Start taking ONS before meals: after breakfast or lunch, patients want to go walking or shopping and want to drink after that. In other words, they want to start drinking the supplement before lunch or dinner. In fact, many patients were taking ONS in this way simply on the advice of their physician to take ONS twice a day. Although the patient's daytime behavior is not restricted, lunch and dinner begin when the blood glucose rises due to taking ONS. The patients complained that they could not eat lunch and dinner because they were full. (3) Start taking ONS at the same time as breakfast and dinner: the start of breakfast and dinner are not times of elevated blood glucose. Moreover, taking ONS at these times does not restrict the behavior of patients during the day. Patients do not suffer because they do not eat in the middle of the night even if their blood sugar rises at bedtime. Additionally, protein intake before sleep is still beneficial in that it is thought to increase muscle protein synthesis ${ }^{[13]}$.

On the basis of these measures, we retrospectively examined the perioperative results of subtotal gastric reconstruction, postoperative body weight change, and quality of life.

\section{Statistical analysis}

Results are expressed as median (interquartile range) or mean (standard deviation) for quantitative variables and percentage for qualitative variables. The $\chi^{2}$ test or Fisher's exact test was used for categorical variables, and the nonparametric Wilcoxon rank sum test was used for continuous variables. All statistical analyses were performed with the SPSS 27.0 software package (SPSS). 


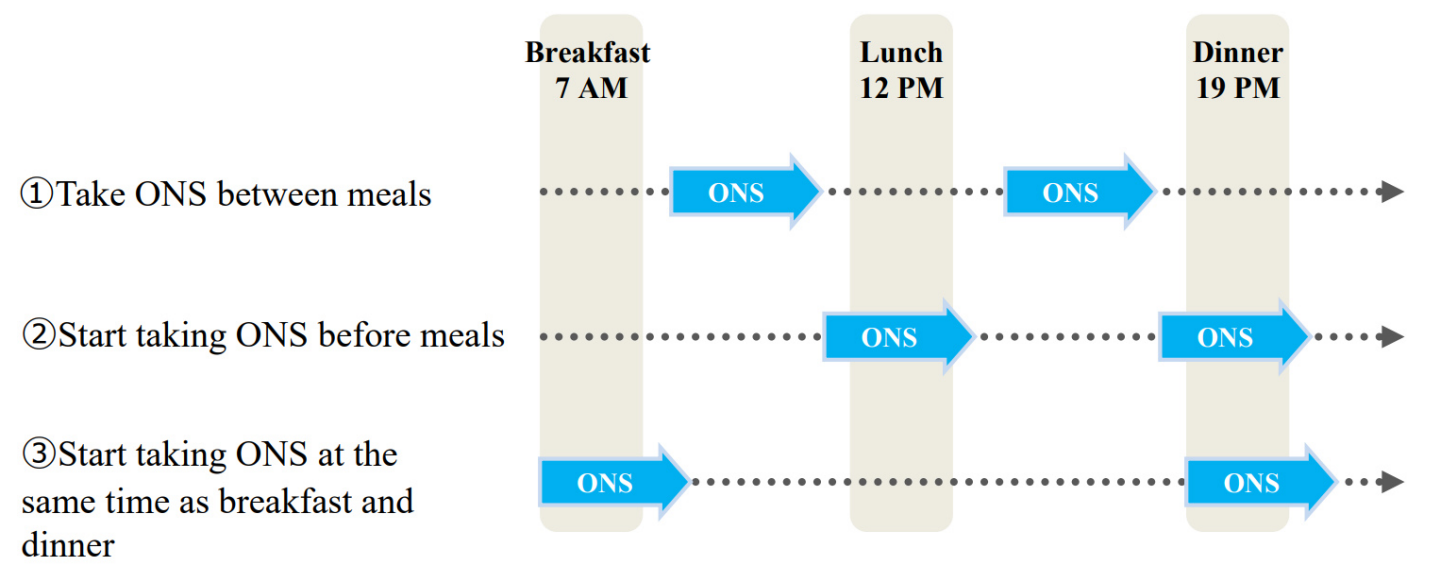

Figure 2. Example of three timings of ONS (oral nutritional supplementation).

This study was approved by the Ethics Committee of Gifu University School of Medicine (IRB Numbers: 2019-149, 2019-170). Written informed consent was obtained from all patients in this study.

\section{RESULTS}

The subjects comprised 386 patients who underwent SS reconstruction during EC surgery performed from January 2008 to January 2021 at Gifu University Hospital. Out of the 386 patients, there were 313 (81\%) men and $73(19 \%)$ women whose mean age was $69.6 \pm 9.2$ years. Clinical stage was I in 77 (20\%), II in 61 (16\%), III in 197 (51\%), and IVA in 51 (13\%) patients (TNM Classification of Malignant Tumours, 8 th $\left.\mathrm{ed}^{[14]}\right)$. Tumor location was the upper esophagus in $53(14 \%)$, middle esophagus in $226(59 \%)$, and lower esophagus in 107 (28\%) patients. The route of reconstruction was substernal in $89(23 \%)$ patients and posterior mediastinal in 297 (77\%) patients. Anastomotic leakage occurred in 2 patients (0.5\%). The average distance from the planned anastomotic site of the SS to the pyloric ring was $29.8 \pm 3.5 \mathrm{~cm}$. No patients had complications where the distance could not be reached during the anastomosis to the cervical esophagus.

We examined the changes in body weight of 223 patients who were traceable postoperatively and confirmed to have survived for 5 years without recurrence. The patients' average body weight was as follows: pretreatment, $55.1 \pm 9.75 \mathrm{~kg}$; 1 month postoperatively, $54.9 \pm 9.09 \mathrm{~kg} ; 3$ months postoperatively, $54.5 \pm$ $8.79 \mathrm{~kg} ; 6$ months postoperatively, $54.1 \pm 8.84 \mathrm{~kg} ; 12$ months postoperatively, $53.9 \pm 8.81 \mathrm{~kg} ; 24$ months postoperatively, $53.6 \pm 8.79 \mathrm{~kg} ; 36$ months postoperatively, $53.4 \pm 8.91 \mathrm{~kg} ; 48$ months postoperatively, $52.9 \pm$ $9.09 \mathrm{~kg}$; and 60 months postoperatively, $52.2 \pm 8.96 \mathrm{~kg}$. The rates of body weight change compared to the first visit were as follows: 1 month postoperatively, $+2.4 \%$; 3 months postoperatively, $-0.51 \%$; 6 months postoperatively, $-1.05 \% ; 12$ months postoperatively, $-1.46 \% ; 24$ months postoperatively, $-2.00 \%$; 36 months postoperatively, $-2.42 \%$; 48 months postoperatively, $-3.39 \%$, and 60 months postoperatively, $-4.78 \%$ [Figure 3].

Early feeling of fullness was assessed using the esophageal site-specific module of the validated European Organization for Research and Treatment of Cancer Quality of Life Questionnaire (EORTC QLQ-OES18) ) $^{[15]}$ at postoperative months $3,12,24,36,48$, and 60 , and the average scores were $1.7 \pm 0.3,2.0 \pm 0.2,1.2 \pm 0.3$, $1.3 \pm 0.2,1.5 \pm 0.2$, and $1.4 \pm 0.1$, respectively. The rate of re-admission due to the delay of gastric emptying was $1.3 \%$. 


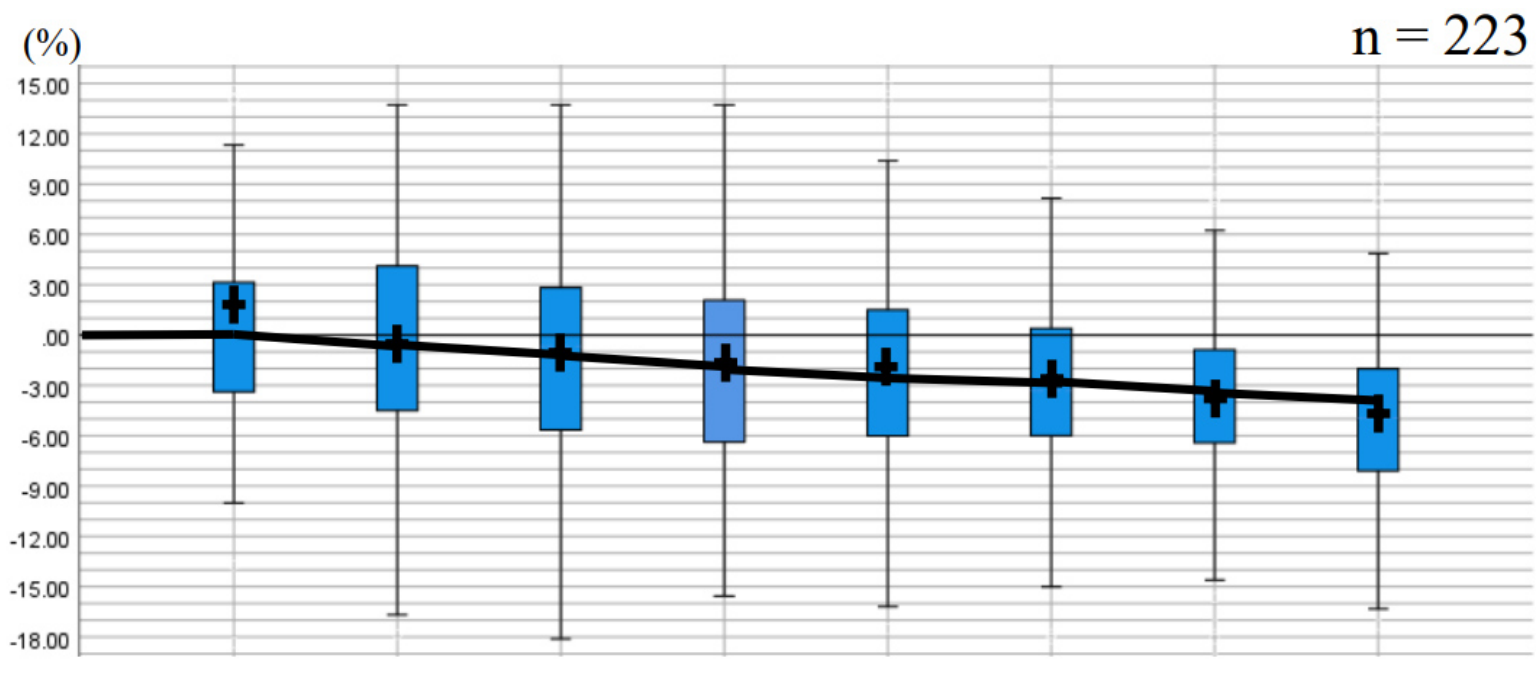

Postoperative 1 month 3 months 6 months 12 months 24 months 36 months 48 months 60 months

$$
\begin{array}{llllllll}
\text { Mean rate of body weight change rate compared to the first visit } \\
+2.4 \% & -0.51 \% & -1.05 \% & -1.46 \% & -2.00 \% & -2.42 \% & -3.39 \% & -4.78 \%
\end{array}
$$

\section{† Mean value}

Line: Median transition

Figure 3. Body weight change after surgery.

\section{DISCUSSION}

A recent review article reported a frequency of anastomotic leakage associated with esophagectomy of $11.4 \%^{[16]}$. Familiarity with the techniques of subtotal gastric reconstruction can lead to a situation that is extremely safe and does not interfere with oral intake. In our department, the phase of development of our technique aimed at reducing anastomotic leakage has ended, and we are now focusing particularly on how to create a reconstructed stomach without causing postoperative difficulty in eating.

It was reported in a systematic review and meta-analysis that there was no difference in delayed gastric emptying with gastric-tube $v s$. whole-stomach ${ }^{[17]}$. SS is close to the whole-stomach, and we think that it is possible to achieve gastric emptying similar to reconstruction using a narrow gastric tube if we are aware of linearization in the thoracic cavity by our method.

It has been suggested that reducing postoperative infectious complications tends to reduce the recurrence of cancer $^{[18]}$, whereas weight loss of $10 \%$ or more was reported to increase the rate of recurrence ${ }^{[19]}$. First, a reduction in the rate of anastomotic leakage significantly helps to suppress the perioperative inflammatory cytokines that trigger the recurrence of cancer metastasis. Second, a surgical reconstruction must be devised that allows smoother flow of oral intake. Schandl et al. ${ }^{[20]}$ reported that among 351 patients, 125 (36\%) suffered from $\geq 15 \%$ postoperative weight loss within 6 months of EC surgery.

There are neurons in the centers of satiety and feeding in the brain that respond to dietary signals by increasing or decreasing blood glucose ${ }^{[21]}$. If the glucose level remains high over a long period of time, caloric intake tends to decrease. Ingestion of $300 \mathrm{kcal}$ significantly reduces appetite when checked at 15, 30, and $60 \mathrm{~min}$ after ingestion ${ }^{[22]}$. In our institution, ONS is administered via jejunostomy tube or orally twice a 


$$
\mathrm{n}=198
$$

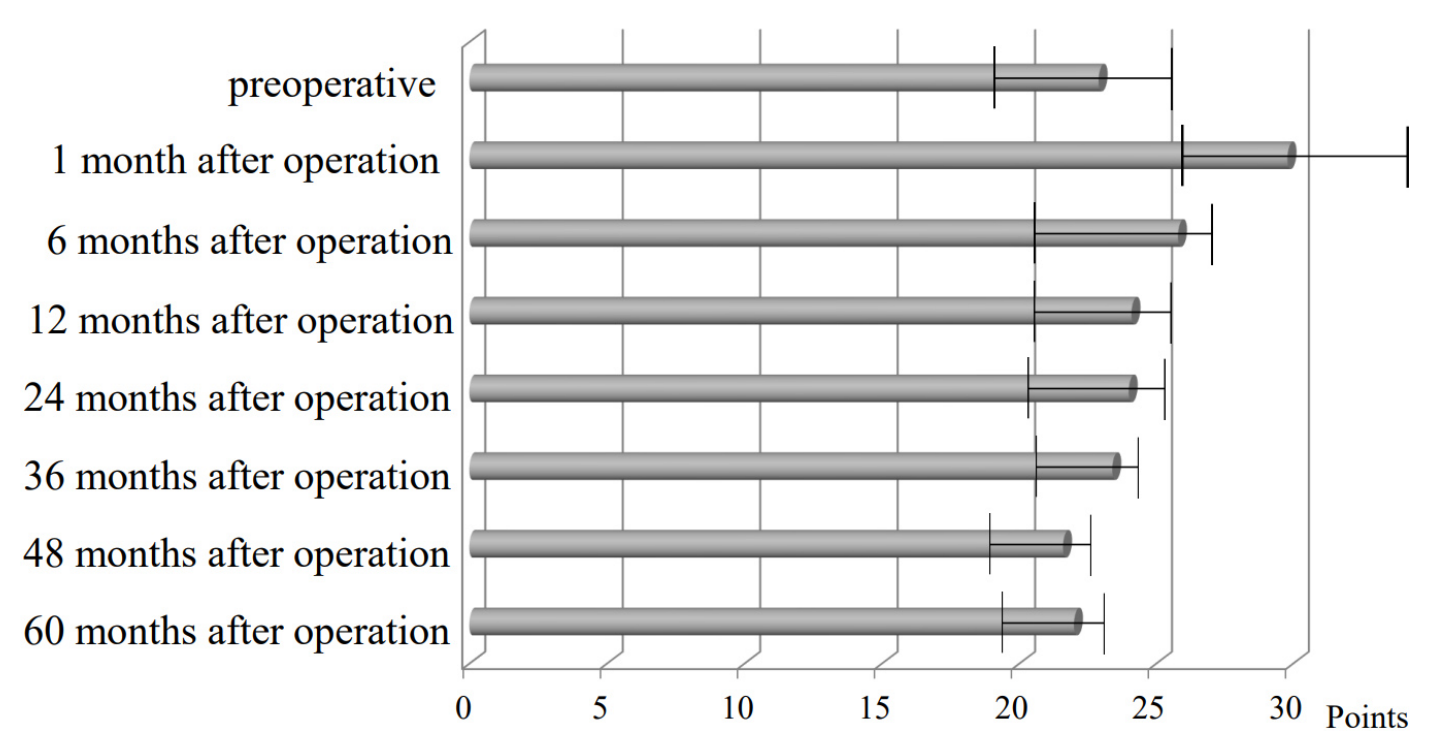

Figure 4. EORTC QLQ-OES18 scoring. EORTC QLQ-OES-18: The European organization for research and treatment of cancer quality of life questionnaire C30 (EORTC QLQ-30) and supplemental esophageal cancer-specific questionnaires.

day at the same time as breakfast and dinner, and an administration time of $3 \mathrm{~h}$ at each meal is strictly adhered to. Patients' compliance with ONS has improved with this timing. We use ENSURE $\mathrm{H}$, which is the concentrated type $(1.5 \mathrm{kcal} / \mathrm{mL})$. Because the ENSURE ${ }^{\oplus} \mathrm{H}$ is taken with meals, it is diluted by mixing with digestive juices in the digestive tract, and its administration is rarely interrupted due to diarrhea unless lactose intolerance is present. We did not set targeted calorie goals in each patient. Even if we set a targeted calorie goal, most patients cannot reach it 1-6 months after EC surgery with a normal diet alone. However, if the shortage is covered completely by ONS, the normal amount of daily food will not increase. So, all the patients received twice a day ( $375 \mathrm{kcal}$ each) as ONS.

By reconstructing a gastric tube that causes no postoperative difficulty in eating as described above and using ONS based on scientific evidence, we have succeeded in limiting the decrease in weight in our patients to $-4.78 \%$ over 5 years. It has been reported that even healthy people lose $-6.5 \%$ in 5 years in their $70 \mathrm{~s}^{[23]}$. Given that, we believe that our postoperative data for EC is not bad at all. The ESPEN 2020 guidelines assert the importance of early perioperative enteral nutritional management, but there is no evidence of how long it should last ${ }^{[24]}$. As a result of examining the cases in our institution regarding esophageal-specific function and symptoms ${ }^{[15]}$, the total scores tended to increase until about half a year after the operation [Figure 4]. Therefore, it is considered feasible to use ONS for half a year after surgery. In fact, our method succeeded in suppressing the weight loss in the 6 months after surgery to $-1.05 \%$.

Recently, we have been using mobile phones to access a one-on-one, completely private social network system [Medical care station (Embrace Co., Ltd)] between medical staff (i.e., doctors, pharmacists, nutritionists, speech therapists, and physiotherapists) and patients as elderly patients may forget our guidance unless they reconfirm the ONS intake method via the social network system. In fact, $98.4 \%$ of patients were able to perform ONS as planned. In addition, not only nutritionists but also physiotherapists participate because improving both swallowing and gastric tube excretion through appropriate exercises leads to better oral intake. Thus, it seems to be important to avoid the marked weight loss for half a year 
after surgery by taking ONS, undergoing gastric tube reconstruction, and allowing for continuous intervention. Regarding the best timing of ONS administration after EC, future large-scale randomized trials are desired.

A meta-analysis did not show the superiority of ONS and reported the benefits of home enteral nutrition ${ }^{[25]}$. This suggests the difficulty of ONS compliance. Several reports showed that ONS did not contribute to weight maintenance ${ }^{[2,2,2]}$. In other words, even if the benefit of ONS is suggested as an underlying mechanism, the results may be affected by the difference between the planned duration of ONS and compliance itself. In that respect, the ONS intake method we reported may be useful in these studies in the future.

This study has several limitations. Our examination of body weight changes focuses on the patients without postoperative recurrence. Novel nutritional management is desired considering cancer metabolism or feasibility of the high caloric supplementation for recurrent EC cases.

Considering different ways to eliminate the factors that prevent continuation of treatment may lead to sustainable treatment against EC. Proper surgical reconstruction and continuous nutritional management with proper guidance can help to maintain body weight and a good quality of life after surgery.

\section{DECLARATIONS}

\section{Acknowledgments}

We would like to thank Rise Japan LLC for providing editorial assistance.

\section{Authors' contributions}

Contributions to conception and design of the study and performed data analysis and interpretation: Tanaka Y, Sato Y, Suetsugu T, Mase J, Takaha R, Okumura N, Matsuhashi N

Performed data acquisition and provided administrative and technical support: Takahashi T, Yoshida K

\section{Availability of data and materials}

Not applicable.

\section{Financial support and sponsorship}

None.

\section{Conflicts of interest}

Dr. Yoshida reports grants, personal fees, and non-financial support from EA Pharma Co., Ltd., Sanofi, Yakult Honsha Co., Ltd., Chugai Pharma Co., Ltd., Taiho Pharma Co., Ltd., Takeda Pharma Co., Ltd., Eli Lilly Japan K.K., Daiichi Sankyo Co., Ltd., Ono Pharma Co., Ltd., Merck Serono Co., Ltd., and Novartis Pharma K.K., and grants from Kyowa Hakko Kirin Co., Ltd. outside the submitted work. Other authors declared that there are no conflicts of interest.

\section{Ethical approval and consent to participate}

This study was conducted in accordance with the World Medical Association Declaration of Helsinki. This study was approved by the Ethics Committee of Gifu University School of Medicine (IRB Numbers: 2019149, 2019-170). 


\section{Consent for publication}

Written informed consent for this study was obtained from all patients.

\section{Copyright}

(c) The Author(s) 2021.

\section{REFERENCES}

1. National Cancer Center Cancer Information Service 2020. Available from: https://ganjoho.jp/en/public/statistics/short pred.html. [Last accessed on 30 Sep 2021].

2. Kelly RJ. Emerging multimodality approaches to treat localized esophageal cancer. J Natl Compr Canc Netw 2019;17:1009-14. DOI PubMed

3. Vellayappan BA, Soon YY, Ku GY, Leong CN, Lu JJ, Tey JC. Chemoradiotherapy versus chemoradiotherapy plus surgery for esophageal cancer. Cochrane Database Syst Rev 2017;8:CD010511. DOI PubMed PMC

4. Tanaka Y, Yoshida K, Sanada Y, Osada S, Yamaguchi K, Takahashi T. Biweekly docetaxel, cisplatin, and 5-fluorouracil (DCF) chemotherapy for advanced esophageal squamous cell carcinoma: a phase I dose-escalation study. Cancer Chemother Pharmacol 2010;66:1159-65. DOI PubMed PMC

5. Tanaka Y, Yoshida K, Yamada A, et al. Phase II trial of biweekly docetaxel, cisplatin, and 5-fluorouracil chemotherapy for advanced esophageal squamous cell carcinoma. Cancer Chemother Pharmacol 2016;77:1143-52. DOI PubMed PMC

6. Tanaka Y, Yoshida K, Osada S, Yamaguchi K, Takahashi T. Docetaxel, nedaplatin, and S-1 (DGS) chemotherapy for advanced esophageal carcinoma: a phase I dose-escalation study. Anticancer Res 2011;31:4589-97. PubMed

7. Tanaka Y, Yoshida K, Tanahashi T, Okumura N, Matsuhashi N, Yamaguchi K. Phase II trial of neoadjuvant chemotherapy with docetaxel, nedaplatin, and S1 for advanced esophageal squamous cell carcinoma. Cancer Sci 2016;107:764-72. DOI PubMed PMC

8. Watanabe M, Otake R, Kozuki R, et al. Recent progress in multidisciplinary treatment for patients with esophageal cancer. Surg Today 2020;50:12-20. DOI PubMed PMC

9. Tanaka Y, Takahashi T, Yamaguchi K, Osada S, Shimokawa T, Yoshida K. Elemental diet plus glutamine for the prevention of mucositis in esophageal cancer patients receiving chemotherapy: a feasibility study. Support Care Cancer 2016;24:933-41. DOI PubMed PMC

10. Tanaka Y, Ueno T, Yoshida N, et al. The effect of an elemental diet on oral mucositis of esophageal cancer patients treated with DCF chemotherapy: a multi-center prospective feasibility study (EPOC study). Esophagus 2018;15:239-48. DOI PubMed PMC

11. Tanaka Y, Ueno T, Yoshida N, et al. Is oral mucositis occurring during chemotherapy for esophageal cancer patients correctly judged? Anticancer Res 2019;39:4441-8. DOI PubMed

12. Yoshida K, Tanaka Y, Imai T, et al. Subtotal stomach in esophageal reconstruction surgery achieves an anastomotic leakage rate of less than 1\%. Ann Gastroenterol Surg 2020;4:422-32. DOI PubMed PMC

13. Kouw IW, Holwerda AM, Trommelen J, et al. Protein ingestion before sleep increases overnight muscle protein synthesis rates in healthy older men: a randomized controlled trial. J Nutr 2017;147:2252-61. DOI PubMed

14. Brierley JD, Gospodarowicz MK, Wittekind C. TNM classification of malignant tumours. 8th ed. Chichester: John Wiley \& Sons, Ltd; 2017.

15. Blazeby J, Conroy T, Hammerlid E, et al. Clinical and psychometric validation of an EORTC questionnaire module, the EORTC QLQOES18, to assess quality of life in patients with oesophageal cancer. Eur J Cancer 2003;39:1384-94. DOI PubMed

16. Ozawa S, Koyanagi K, Ninomiya Y, Yatabe K, Higuchi T. Postoperative complications of minimally invasive esophagectomy for esophageal cancer. Ann Gastroenterol Surg 2020;4:126-34. DOI PubMed PMC

17. Zhang W, Yu D, Peng J, Xu J, Wei Y. Gastric-tube versus whole-stomach esophagectomy for esophageal cancer: a systematic review and meta-analysis. PLoS One 2017;12:e0173416. DOI PubMed PMC

18. Beecher SM, O’Leary DP, McLaughlin R, Kerin MJ. The impact of surgical complications on cancer recurrence rates: a literature review. Oncol Res Treat 2018;41:478-82. DOI PubMed

19. D'Journo XB, Ouattara M, Loundou A, et al. Prognostic impact of weight loss in 1-year survivors after transthoracic esophagectomy for cancer. Dis Esophagus 2012;25:527-34. DOI PubMed

20. Schandl A, Kauppila JH, Anandavadivelan P, Johar A, Lagergren P. Predicting the risk of weight loss after esophageal cancer surgery. Ann Surg Oncol 2019;26:2385-91. DOI PubMed PMC

21. Oomura Y, Ono T, Ooyama H, Wayner MJ. Glucose and osmosensitive neurones of the rat hypothalamus. Nature 1969;222:282-4. DOI PubMed

22. Anderson GH, Woodend D. Consumption of sugars and the regulation of short-term satiety and food intake. Am $J$ Clin Nutr 2003;78:843S-9S. DOI PubMed

23. Yonei Y, Miwa Y, Hibino S, et al. Japanese anthropometric reference data - special emphasis on bioelectrical impedance analysis of muscle mass. Anti-Aging Med 2008;5:63-72. DOI

24. Lobo DN, Gianotti L, Adiamah A, et al. Perioperative nutrition: recommendations from the ESPEN expert group. Clin Nutr 2020;39:3211-27. DOI PubMed

25. Xueting H, Li L, Meng Y, et al. Home enteral nutrition and oral nutritional supplements in postoperative patients with upper gastrointestinal malignancy: a systematic review and meta-analysis. Clin Nutr 2021;40:3082-93. DOI PubMed 
26. Ida S, Hiki N, Cho H, et al. Randomized clinical trial comparing standard diet with perioperative oral immunonutrition in total gastrectomy for gastric cancer. Br J Surg 2017;104:377-83. DOI PubMed

27. Aoyama T, Yoshikawa T, Ida S, et al. Effects of perioperative Eicosapentaenoic acid-enriched oral nutritional supplement on lean body mass after total gastrectomy for gastric cancer. J Cancer 2019;10:1070-6. DOI PubMed PMC 